\title{
Exactly Solvable Model of a Superconducting to Rotational Phase Transition
}

\author{
D. J. Rowe, C. Bahri, and W. Wijesundera \\ Department of Physics, University of Toronto, Toronto, Ontario, Canada M5S 1A7
}

(Received 29 August 1997)

\begin{abstract}
We consider a many-fermion model which exhibits a transition from a superconducting to a rotational phase with variation of a parameter in its Hamiltonian. The model has analytical solutions in its two limits due to the presence of dynamical symmetries. However, the symmetries are basically incompatible with one another; no simple solution exists in intermediate situations. Exact (numerical) solutions are possible and enable one to study the behavior of competing but incompatible symmetries and the phase transitions that result in a semirealistic situation. The results are remarkably simple and shed light on the nature of phase transitions. [S0031-9007(98)05989-4]

PACS numbers: 21.60.Fw, 03.65.Fd, 05.70.Fh, 21.60.Ev
\end{abstract}

Much can be learned about the validity of approximate many-body theories by applying them to exactly solvable models $[1,2]$. We consider a model with two simple limits (phases): one superconducting and the other rotational. Systems of this kind are prevalent in nuclear physics. Indeed, most open-shell nuclei are regarded as being vibrational and superconducting or deformed and rotational. Some nuclei appear to be both rotational and superconducting. Thus, we consider a model with Hamiltonian

$$
H=\alpha H_{1}+(1-\alpha) H_{2},
$$

where $H_{1}$ and $H_{2}$ are, respectively, Hamiltonians for rotational motion and superconductivity; $\alpha$ is a parameter in the range $0 \leq \alpha \leq 1$. With variation of $\alpha$, the system undergoes a transition from one phase to the other.

A common approach to understanding such systems is with the Hartree-Fock-Bogolyubov (HFB) approximation or one of its phenomenological variations. Such mean-field theories yield many solutions in general: some spherical and superconducting and others deformed and rotational. Moreover, with variation of the particle number or parameters in the Hamiltonian, phase transitions occur in the solution which lies lowest in energy. This motivates one to inquire if the phase transitions are real or are artifacts of the HFB constraints. When improved approximations are made, e.g., by restoring broken rotational and particle number symmetries by angular momentum and number projection, it appears that the phase transitions are smoothed out [3]. Remnants may survive and even become sharp in large systems. But, one needs to compare the results with exact calculations, to know what they have to say about physical phase transitions.

Exact results for truncated spaces can be obtained with a shell model code. For example, Bahri et al. [4] have investigated the competition between superconductivity and rotational dynamics for a system of identical nucleons in the nuclear $f p$ shell. The remarkable fact is that superconductivity and rotations, separately, can be treated rather well as a result of their dynamical symmetries [5]. However, for a combination of the two, one needs a huge space to include all the relevant correlations. This is because the dynamical symmetries for rotations and superconductivity are incompatible according to the following definition [6].

If a Hilbert space $\mathbb{W}$ carries reducible representations of two groups, the actions of the two groups are said to be incompatible if they do not commute with one another and if $\mathbb{W}$ has no proper subspace that is invariant under both groups. For example, two Lie group actions might be incompatible because there exists no finite-dimensional Lie group that contains both groups as subgroups.

Microscopic models for the rotational states of an $A$ particle nucleus are given by Hamiltonians of the form

$$
H_{\mathrm{RM}}=\frac{1}{2 m} \sum_{n}^{A} p_{n}^{2}+\frac{1}{2} m \omega^{2} \sum_{n}^{A} r_{n}^{2}+V(Q),
$$

where $V(Q)$ is a low order polynomial in the quadrupole moments of the nucleus [7]. The building blocks of such a Hamiltonian $\left(\sum p_{n}^{2}, \sum r_{n}^{2}\right.$, and the components of $Q)$ are elements of the (noncompact) symplectic algebra $\mathrm{Sp}(3, \mathbb{R})$. Thus, $H_{\mathrm{RM}}$ is diagonalizable on a computer (to within round-off errors).

Models of superconductivity [8] are given by Hamiltonians of the form

$$
H_{\mathrm{SC}}=\sum \varepsilon_{j} a_{j m}^{\dagger} a_{j m}-\sum G_{j j^{\prime}} a_{j m}^{\dagger} a_{j \bar{m}}^{\dagger} a_{j^{\prime} \bar{m}^{\prime}} a_{j^{\prime} m^{\prime}}
$$

where $\left\{a_{j m}^{\dagger}, a_{j m}\right\}$ are creation and annihilation operators for fermions of angular momentum $j$ and $z$ component $m$; $j \bar{m}$ label the time reverse of the state labeled by $j m$. Such a Hamiltonian has a spectrum generating algebra (SGA) comprising a direct sum of SU(2) (quasispin) algebras [9]. Consequently, it can be diagonalized numerically (if the number of single-particle states is not too large) [10].

The actions of the $\operatorname{Sp}(3, \mathbb{R})$ and quasispin Lie algebras are incompatible. As a result, it is not possible to diagonalize a linear combination of $H_{\mathrm{RM}}$ and $H_{\mathrm{SC}}$ without an unjustified truncation of the space. It is then an interesting challenge to find approximate methods for determining the spectrum of such combinations. 
To simplify the problem, we restrict the symplectic model to a single spherical harmonic oscillator shell. The model then reduces to Elliott's model [11] for which a suitable Hamiltonian is

$$
H_{1}=-\chi \mathcal{Q} \cdot \mathcal{Q},
$$

where $\mathcal{Q}$ is the $\mathrm{SU}(3)$ quadrupole tensor. This Hamiltonian has spectrum given by

$$
E(\lambda \mu J)=-\chi C_{2}(\lambda \mu)+3 \chi J(J+1),
$$

where $(\lambda \mu)$ is an $\mathrm{SU}(3)$ highest weight, $J$ is the angular momentum, and

$$
C_{2}(\lambda \mu)=4\left(\lambda^{2}+\mu^{2}+\lambda \mu+3 \lambda+3 \mu\right)
$$

is the value of the $\mathrm{SU}(3)$ Casimir invariant.

A simplification of $H_{\mathrm{SC}}$ is obtained by putting all single-particle energies equal and setting $G_{j j^{\prime}}=G$. The Hamiltonian $H_{\mathrm{SC}}$ then reduces to

$$
H_{2}=-G S_{+} S_{-},
$$

where

$$
\begin{aligned}
& S_{+}=\sum_{j m>0}(-1)^{j+m} a_{j m}^{\dagger} a_{j,-m}^{\dagger}, \\
& S_{-}=\sum_{j m>0}(-1)^{j+m} a_{j,-m} a_{j m} .
\end{aligned}
$$

This Hamiltonian has analytical solutions because, as shown by Kerman et al. [9], $S_{ \pm}$are the raising and lowering operators of a single $\mathrm{SU}(2)$ algebra with $\left[S_{+}, S_{-}\right]=$ $2 S_{0}$ and $\left[S_{0}, S_{ \pm}\right]= \pm S_{ \pm}$. It has a spectrum given by

$$
E(N, s)=-G s(s+1)+\frac{1}{4} G(N-2 s)(N-2 s-2) \text {. }
$$

With these simplifications, the dynamical symmetries of $H_{1}$ and $H_{2}$ can be combined. The smallest Lie algebra that contains both $\mathrm{SU}(2)$ and $\mathrm{SU}(3)$, acting within the space of a many-particle spherical harmonic oscillator shell, is the compact symplectic algebra $\operatorname{Sp}(n)$; an algebra of rank $n$ equal to the number, $n=\frac{1}{2}(\nu+1)(\nu+2)$, of orbital single-particle states for the shell with $\nu$ harmonic oscillator quanta. It is spanned by operators

$$
\begin{aligned}
A_{i j} & =\sum_{k m}(-1)^{(1 / 2)+m} a_{k i m}^{\dagger} a_{k j,-m}^{\dagger}, \\
B_{i j} & =\sum_{k m}(-1)^{(1 / 2)+m} a_{k i m} a_{k j,-m}, \\
C_{i j} & =\frac{1}{2} \sum_{k m}\left(a_{k i m}^{\dagger} a_{k j m}-a_{k j m} a_{k i m}^{\dagger}\right),
\end{aligned}
$$

where $m$ is summed over spin values $\pm 1 / 2, i$ and $j$ run over the range $1, \ldots, \nu$, and $k$ is summed over $\kappa$ fermion types; e.g., $\kappa=2$ for neutrons and protons.

For $n \geq 3, \mathrm{Sp}(n)$ contains Elliott's SU(3) and Kerman's $\mathrm{SU}(2)$ quasispin algebra, with

$$
\begin{gathered}
S_{+}=\frac{1}{2} \sum_{i} A_{i i}, \quad S_{0}=\frac{1}{2} \sum_{i} C_{i i}, \\
S_{-}=\frac{1}{2} \sum_{i} B_{i i},
\end{gathered}
$$

as subalgebras. Thus, $\operatorname{Sp}(n)$ is a SGA for the Hamiltonian of Eq. (1). We consider here the smallest $\mathrm{Sp}(n)$ Lie algebra that contains both $\mathrm{SU}(3)$ and $\mathrm{SU}(2)$, i.e., $\mathrm{Sp}(3)$. One recalls that $\mathrm{SO}(5)$ symmetry [isomorphic to $\mathrm{Sp}(2)$ ] has been used by Zhang and Demler [12] to explore the competition between antiferromagnetism and $d$-wave superconductivity within the framework of the Hubbard model.

The relevant irrep of $\mathrm{Sp}(3)$, for present purposes, is the one whose lowest weight state is the fermion vacuum state $|0\rangle$. This state satisfies the equations

$$
B_{i j}|0\rangle=0, \quad C_{i j}|0\rangle=-\delta_{i j} \kappa|0\rangle,
$$

which means that it is a lowest weight state for an $\mathrm{Sp}(3)$ irrep with weight $(-\kappa,-\kappa,-\kappa)$.

Basis states for an irrep with this lowest weight are generated by repeated application of the $S p(3)$ raising operators $\left\{A_{i j}\right\}$ to the vacuum state $|0\rangle$. These operators transform as a basis for a $\mathrm{U}(3)$ irrep of highest weight $\{200\}$. Thus, an orthonormal basis $\{|n K J M\rangle\}$ for the desired $\mathrm{Sp}(3)$ irrep can be constructed, which reduces the subalgebra chain

$$
\mathrm{Sp}(3) \supset \mathrm{U}(3) \supset \mathrm{SU}(3) \supset \mathrm{SO}(3) \supset \mathrm{SO}(2),
$$

where $n \equiv\left\{n_{1}, n_{2}, n_{3}\right\}$, a triple of integers with $n_{1} \geq$ $n_{2} \geq n_{3}$, is a $\mathrm{U}(3)$ highest weight and $J M$ are the usual $\mathrm{SO}(3) \supset \mathrm{SO}(2)$ angular momentum quantum numbers; $K$ indexes multiplicities of $J$ in the SU(3) irrep with highest weight $(\lambda, \mu)=\left(n_{1}-n_{2}, n_{2}-n_{3}\right)$.

The matrices of such irreps of the symplectic Lie algebras are known [13,14]; explicit expressions will be given in a more complete report to follow.

For $\kappa=2$, one obtains $\operatorname{Sp}(3)$ representations appropriate for two kinds of nucleon (neutrons and protons) filling the $1 p$ shell. This space is too small; the most deformed $\mathrm{SU}(3)$ irrep it contains is of highest weight $(\lambda \mu)=(4,0)$, whereas the irreps relevant for the description of rotational bands in rare-earth nuclei [15] have $\lambda \approx 70-100$, $\mu \approx 0-10$. Such SU(3) irreps are found in the nuclear shell model for two kinds of nucleon (i.e., $\kappa=2$ ) filling the $\nu=4$ and 5 shells. However, mixed SU(3) calculations in these shells are impossible. We therefore generate the desired SU(3) irreps in our model, in which the most deformed SU(3) irrep is of highest weight $(\lambda \mu)=(2 \kappa, 0)$, by artificially assigning $\kappa$ values in the 20-40 range.

We stress that our objective in this paper is not to fit any particular data. It is rather to construct an exactly solvable model that can be used to test ideas and many-body theories. It should also be understood that our model can be derived in many ways. For example, one can regard its $\mathrm{Sp}(3)$ algebra as a subalgebra of a larger $\mathrm{Sp}(n)$ Lie algebra using pseudospin ideas following Ginocchio [16]. In selecting $\mathrm{Sp}(3)$, we have been guided by experience 
with the noncompact $\operatorname{Sp}(3, \mathbb{R})$ algebra [7], the interacting boson model [17], and the Fermion dynamical symmetry model [18]. In fact, our model contracts to the interacting boson model as $\kappa \rightarrow \infty$.

Results for the excitation energies of the lowest $J=0$, 2, 4, 6, and 8 states are plotted in Fig. 1 for $N=2 \kappa$, $\kappa=5,10$, and 20, and coupling constants $G=0.05$ and $\chi=0.005$ (dimensionless units). The $N / \kappa=2$ ratio corresponds to selecting the same one-third-filled shell nucleus in each case. The remarkable feature of the results is the sharpness of the transition from the superconducting to the rotational phase. For all $\kappa$ values shown, the system is essentially in a superconducting phase for $\alpha<0.5$ and a rotational phase for $\alpha>0.6$. The figure also shows the expectation values $\left\langle S_{+} S_{-}\right\rangle_{\alpha}$ and $\left\langle C_{2}\right\rangle_{\alpha}$ as functions of $\alpha$ for each $J$, where $C_{2}$ is the $\mathrm{SU}(3)$ Casimir operator. The results exhibit the extraordinary purity of the wave functions on either side of the phase transition. For large $\kappa$ values, $\left\langle S_{+} S_{-}\right\rangle_{\alpha}$ remains close to its $\alpha=0$ value until $\alpha$ approaches a critical value $\alpha_{0}$. For $\alpha>\alpha_{0},\left\langle S_{+} S_{-}\right\rangle_{\alpha}$ falls rapidly to a small $J$-independent value. Similarly, $\left\langle C_{2}\right\rangle_{\alpha}$ holds closely to its $\alpha=1$ value until $\alpha$ approaches $\alpha_{0}$ from above after which it rapidly falls to a set of equally spaced values for each $J$.

The purity of the wave functions is also indicated by the closeness of the results for the excitation energies to what one would get in first order perturbation theory starting from the appropriate limit. Thus, if one determines energy levels to first order in $\alpha$, one obtains

$$
\begin{aligned}
E_{J}(\alpha)= & E_{J}(0) \\
& +\alpha\left[G\left\langle S_{+} S_{-}\right\rangle_{0}-4 \chi\left\langle C_{2}\right\rangle_{0}+3 \chi J(J+1)\right] .
\end{aligned}
$$
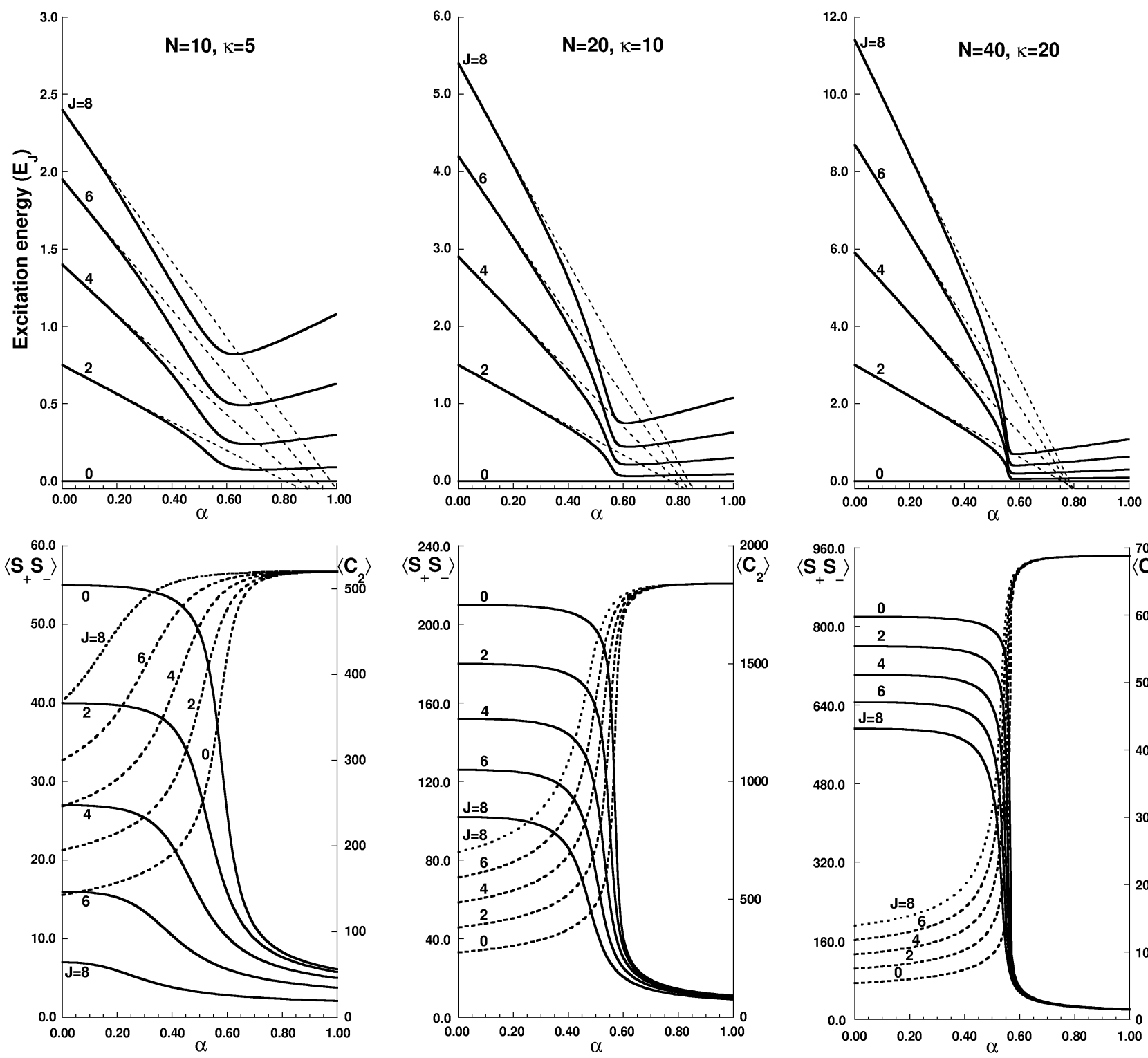

FIG. 1. The upper figures show the excitation energies for the Hamiltonian of Eq. (1) for different values of $\kappa$ and $N=2 \kappa$. The lower figures show the expectations $\left\langle S_{+} S_{-}\right\rangle$and $\left\langle C_{2}\right\rangle$ as functions of $\alpha$ for the corresponding $\kappa$ values. 
The linear expressions for the excitation energies, shown in the figure, indicate that the exactly computed results only diverge substantially from the first order expressions when $\alpha$ is relatively close to $\alpha_{0}$. On the other hand, if one determines energy levels to first order in $(1-\alpha)$,

$$
\begin{aligned}
E_{J}(\alpha)= & E_{J}(1) \\
& -(1-\alpha)\left[G\left\langle S_{+} S_{-}\right\rangle_{1}-4 \chi\left\langle C_{2}\right\rangle_{1}-3 \chi J(J+1)\right] .
\end{aligned}
$$

The way in which the vibrational excitation energies drop precipitously just prior to the phase transition are reminiscent of RPA (random phase approximation) results for quadrupole vibrational excitation energies. As shown by Thouless [19], within the framework of the RPA, the collapse of a vibrational energy to zero indicates an instability of the model ground state against vibrational fluctuations and the expectation of a phase transition to a deformed state. The present model, having a fermion foundation is clearly amenable to analyses along quasiparticle RPA lines.

It is noteworthy that, for large $\kappa$ and $N=2 \kappa$, there is essentially no region of $\alpha$ for which the model is both rotational and superconducting. It appears that once the system starts to exhibit rotational states, the superconducting pair correlations are quickly suppressed. One recalls the similarity, noted by Mottelson and Valatin [20], between the Coriolis force acting on a particle in a rotating frame of reference and the interaction of a charged particle with a magnetic field. One knows that superconductivity tends to be excluded by a magnetic field; this is the Meissner effect. Mottelson and Valatin suggested that a similar collapse of superconducting pair correlations should be expected in rotational nuclei. However, their expectation was that the collapse would occur above a certain critical angular momentum. Thus, we would not have been surprised to obtain coexisting rotations and superconductivity at low angular momenta. Indeed, one of our motivations for considering the present model was to study superconducting flows in rotational nuclei. Thus, it is of considerable interest to understand what is special about our model that precludes this occurrence. [Subsequent calculations reveal that the strong suppression of superconductivity occurs in our model only when the number of nucleons $N$ is set equal to $2 \kappa$, as in the calculations reported here. We find that, for $N>2 \kappa$, the pair correlations cause a mixing of SU(3) irreps in a highly coherent way such that the rotational character of the states is preserved. Results of these calculations will be reported in the more detailed analysis of the model to follow.]

Outside of a narrow transition region, both phases of the system show a remarkable resilience to being perturbed. This we believe is due, in part, to the fact that the two phases are associated with incompatible dynami- cal symmetries. Put another way, the eigenstates of one phase are spread extremely thinly over the eigenstates of the other. However, consideration of other models indicates that this alone is not sufficient for a sharp phase transition to occur. For example, to see a sharp phase transition, it appears to be important to have many particles but only two-body interactions.

The authors thank Joe Repka for helpful suggestions.

[1] H. J. Lipkin, N. Meshkov, and A. J. Glick, Nucl. Phys. 62, 188 (1965); J. C. Parikh and D. J. Rowe, Phys. Rev. 175, 1293 (1968).

[2] H. Chen, J. Browstein, and D. J. Rowe, Phys. Rev. C 42, 1422 (1990).

[3] P. Ring and P. Schuck, The Nuclear Many-body Problem (Springer, New York, 1980), Chap. 11.

[4] C. Bahri, J. Escher, and J. P. Draayer, Nucl. Phys. A592, 171 (1995).

[5] D. J. Rowe, Prog. Part. Nucl. Phys. 37, 265 (1996).

[6] D. J. Rowe, in New Perspectives in Nuclear Structure, edited by A. Covello (World Scientific, Singapore, 1996).

[7] G. Rosensteel and D. J. Rowe, Phys. Rev. Lett. 38, 10 (1977); Ann. Phys. (N.Y.) 126, 343 (1980); P. Park et al., Nucl. Phys. A414, 93 (1984); D. J. Rowe, Rep. Prog. Phys. 48, 1419 (1985); C. Bahri and D. J. Rowe (to be published).

[8] G. Racah, Phys. Rev. 63, 367 (1943).

[9] A. K. Kerman, Phys. Rev. 12, 300 (1961); A. K. Kerman, R. D. Lawson, and M. W. Macfarlane, Phys. Rev. 124, 162 (1961).

[10] H. Chen, T. Song, and D. J. Rowe, Nucl. Phys. A582, 181 (1995).

[11] J. P. Elliott, Proc. R. Soc. London A 245, 128 (1958); 245, 562 (1958).

[12] S. C. Zhang, Science 275, 1089 (1997); E. Demler and S. C. Zhang, Phys. Rev. Lett. 75, 4126 (1995).

[13] D. J. Rowe, J. Math. Phys. (N.Y.) 25, 2662 (1984); D. J. Rowe, G. Rosensteel, and R. Carr, J. Phys. A 17, L399 (1984); D. J. Rowe and J. Repka, J. Math. Phys. (N.Y.) 32, 2614 (1991).

[14] D. J. Rowe, B. G. Wybourne, and P. H. Butler, J. Phys. A 18, 939 (1985).

[15] M. Jarrio, J. L. Wood, and D. J. Rowe, Nucl. Phys. A528, 409 (1991).

[16] J. N. Ginocchio, Ann. Phys. (N.Y.) 126, 234 (1980).

[17] F. Iachello and A. Arima, The Interacting Boson Model (Cambridge University Press, Cambridge, 1987); D. Bonatsos, Interacting Boson Models of Nuclear Structure (Clarendon, Oxford, 1988).

[18] C-L. Wu, D. H. Feng, and M. Guidry, Adv. Nucl. Phys. 21, 227 (1994).

[19] D. J. Thouless, Nucl. Phys. 21, 225 (1960); D. J. Rowe, Nuclear Collective Motion (Methuen, London, 1970).

[20] B. R. Mottelson and J. G. Valatin, Phys. Rev. Lett. 5, 511 (1960). 